equipment, environment layout, workflow, system processes, effective team training, and ultimately patient safety.

Keywords: simulation, trauma, patient safety

\section{Posters Presentations}

\section{P001}

Concussion patients in the emergency department: assessing a new triaging tool for follow-up and prompt long term management D.A. Abourbih, MD, MSc, S. Bedi, MD, C. Hunt, PhD, D. Ouchterlony, MD, A. Ackery, MD; University of Toronto, Toronto, ON

Introduction: Concussion is a common emergency department (ED) presentation. Most patients improve with expectant management. A subset with risk factors for post-concussion syndrome (PCS) may require closer outpatient follow-up. A novel emergency department (ED)/head injury clinic (HIC) triaging system has been created to allow concussed patients rapid access to educational information and specialized consultant services. This system has been well received by patients and physicians alike; however, objective measures are needed to determine if this system ultimately decreases excessive healthcare utilization (HCU) and improves symptom management of PCS. Methods: Single centered prospective observational study. Control population of $42 \mathrm{mTBI}$ patients referred to the HIC through the Ontario Acquired Brain Injury (ABI) Network within 3-12 months of injury. These patients have received little concussion education or treatment and will be compared to 50 concussion patients seen in the ED and HIC. Rivermead scores, a validated likert scale of PCS symptoms (1-4, maximum score of 64) and HCU (patient reported number of healthcare visits post injury) will be collected on their initial clinic visit and subsequent follow up phone interview. Results: Control ABI network patients were $50 \%$ male, mean age $40 \mathrm{yrs}(18-90, \pm 16.3)$ while $83 \%(35 / 42)$ reported $>1$ subsequent visit to $\mathrm{ED}$ or family physician and 39\% (16/42) visited neurologist. Mean Rivermead Score was 32.6 (7-58, \pm 12 ). Conclusion: A significant proportion of control patients utilized multiple healthcare resources and were still symptomatic 3-6 months following injury. Data collection is currently ongoing to determine if rapid outpatient follow-up and education decreases HCU and PCS symptoms.

Keywords: concussion, triaging tool, Rivermead

\section{P002}

Ten patients, one ventilator: how to best allocate critical care resources during mass disaster

C. Acton, MB, BCh, BAO, M. McGowan, MHK, S.H. Gray, MD; University of Toronto, Toronto, $\mathrm{ON}$

Introduction: Any large-scale disaster may place a hospital system in a precarious position. Planning is fundamental to facilitate an equitable process for allocating scarce critical care resources, yet there is a paucity of literature guiding protocol development, and few Canadian hospitals have done this planning. We performed a scoping review of the available literature, and used this data to develop a hospital-wide policy to guide critical care resource allocation as part of the hospital emergency management planning process. Methods: A primary search of MEDLINE (1946-2015), EMBASE (1980-2015), Disaster Lit (2002-2010) and Pubmed focusing on a priori criteria was completed. A secondary search of the grey literature served to increase sensitivity and rigor. Two independent reviewers manually reviewed the citations, and selected eligible abstracts for full-text. Qualitative thematic analysis was undertaken of the selected articles. The results then informed the development of a hospital-wide policy and protocol to guide critical care resource allocation. Results: The search identified 832 citations; 134 papers were reviewed and 11 selected for qualitative analysis. All included papers were expert opinion and reviews. All suggested that an ethical framework be used; eight discussed this in detail. Ten recommended allocating a triage team to implement the protocol. Nine papers recommended specific resource allocation protocols with inclusion/ exclusion criteria, physiologic scores, and reassessment at varying time intervals (12-120 hours). Conclusion: Effective planning, prior to a disaster, is critical to saving as many lives as possible. Based on our scoping review, we have developed a hospital-wide protocol that incorporates ethical principles and clear inclusion and exclusion criteria, to help avoid inequity and promote transparent decision-making. Next steps include a public consultation process and review, prior to implementation testing and educational roll-out.

Keywords: critical care, mass disaster, policy development

P003

Do all clavicle fractures in children need to be managed by orthopaedic surgeons?

J.S. Adamich, BHSc, M. Camp, MD, MSc, A. Howard, MD, MSc; The Hospital for Sick Children, Toronto, ON

Introduction: Although many uncomplicated pediatric fractures do not require routine long-term follow-up with an orthopedic surgeon, practitioners with limited experience dealing with pediatrics fractures will often defer to a strategy of frequent clinical and radiographic follow-up. Development of an evidence-based clinical care pathway can help unnecessary radiation exposure to this patient population and reduce costs to patient families and the healthcare system. Methods: A retrospective analysis including patients who presented to the Hospital for Sick Children (SickKids) for management of clavicle fractures was performed. Results: Three hundred and forty patients (227 males, 113 females) with an average age of 8.1 (range 0.1-17.8) were included in the study. The mean number of clinic visits including initial consultation in the emergency department was $2.1( \pm 1.3)$. The mean number of radiology department appointments was $1.8( \pm 1.3)$ where patients received a mean number of $4.2( \pm 3.0)$ radiographs. Complications were minimal; 2 refractures in our series and no known cases of non-union. All patients achieved clinical and radiographic union and returned to sport after fracture healing. Conclusion: Our series suggests that the decision to treat operatively is made at the initial assessment. If no surgical indications were present at the initial assessment by the primary-care physician, then routine clinical or radiographic follow up is unnecessary. Our paediatric clavicle fracture pathway will reduce patient radiation exposure and reduce costs incurred by the healthcare system and patients' families without jeopardizing patient outcomes.

Keywords: clavicle fracture, clinical pathway, management

\section{P004}

What happens to cognitive load during trauma skill training using computer based video instructions?

R.N. Addison, MSc, L.E. Rohr, PhD, A. Dubrowski, PhD; Memorial University of Newfoundland, St John's, NL

Introduction: In the clinical settings, emergency physicians are faced with situations that require multitasking such as interacting with other team members, documentation and utilization of computer resources whiles ensuring competency on a particular trauma skill. The purpose of 
this study was to assess the effect of multitasking on cognitive load when learning a common trauma skill. Methods: Sixteen students who had no previous experience with the one-handed square knot were randomly assigned to one of the two groups: practice and non-practice groups. A pre-recorded instructional video of a one-hand knot tying was presented to all participants. Next, the practice group completed a single session of 10 trials of the one-hand knot tying using a benchtop simulator, while the non-practice group did not. All returned a week later for a transfer task on a different simulator. On trials 1, 4, 7,10 and during the transfer performance the participants performed under dual task conditions, where they were asked to focus on the knot-tying task, but also to react as fast as possible to the illumination of an incandescent light bulb by pressing a foot pedal. Subjective ratings of mental effort, and reaction time to the visual stimulus were used as indices of cognitive load. Results: A repeated measure ANOVA showed a significant effect of dual task on subjective measure of mental effort $\left(F_{(4,28)}=10.35, p=.001, \omega=.60\right)$ and reaction time $\left(F_{(4,28)}=\right.$ $7.93, p=.001, \omega=.53$ ), with plots indicating cognitive load plateaued by the $7^{\text {th }}$ trial. Conclusion: These findings highlights the number of trials necessary to attain a level of proficiency in a basic trauma skill such as the one-hand knot tying, ease the level of cognitive load and possibly enhance transfer to more challenging tasks.

Keywords: dual-task, trauma skills, cognitive load

\section{P005 \\ Pre-hospital dexamethasone administration in children with croup: the Edmonton experience}

S. Ali, MDCM, A. Moodley, MD, A. Bhattacharjee, BSc, K. Lobay, DMD, MD, E. Chang, MD, A. Kabaroff, MD, D. Allain, MD; University of Alberta, Edmonton, $\mathrm{AB}$

Introduction: Croup is one of the most common childhood respiratory illnesses, affecting more than 80,000 Canadian children per year. Early dexamethasone administration in croup can reduce admission rates and length of stay (LOS), as well as return visits to the emergency department (ED). Pre-hospital emergency medical services (EMS) teams in Edmonton administer dexamethasone to children with croup. The objectives of this study were to (a) assess the clinical impact of prehospital administration of dexamethasone to children with croup and (b) compare clinical outcomes of these patients to those who did not receive their first dose of dexamethasone via the EMS providers. Methods: This study was a retrospective medical record review that included children between 6 months and 6 years of age who were brought via EMS to the Stollery Children's Hospital ED with a final diagnosis of croup, between January 1st 2010 and December 31 2012. Data were collected regarding pre-hospital presentation and management, ED presentation and management, ED LOS and final disposition, and patient demographics. Results: 188 patients were enrolled, $35.1 \%$ (66/188) of whom received a pre-hospital diagnosis of croup. The mean age of the participants was 32.96 months ( $S D \pm 17.18$ ). Overall, $10.6 \%$ patients (20/188) were given dexamethasone in the pre-hospital setting, while $30.3 \%$ patients $(57 / 188)$ were given nebulized epinephrine by EMS. Out of the 66 patients with a pre-hospital diagnosis of croup, $10.6 \%$ $(\mathrm{n}=7)$ were given dexamethasone by EMS. In the ED, dexamethasone was administered to $88.3 \%$ of patients $(166 / 188)$ while $56 / 188$ participants $(29.8 \%)$ received nebulized epinephrine. There was no statistically significant difference in ED LOS stay between those who received pre-hospital dexamethasone (2.6 hours, $\mathrm{SD} \pm 1.6, \mathrm{n}=18$ ) and those who did not (3.3 hours, $\mathrm{SD} \pm 2.7, \mathrm{n}=159$ ). The number of in-hospital epinephrine doses per patient was influenced by the administration of pre-hospital dexamethasone $(\mathrm{p}=0.010)$. Conclusion: Pre-hospital administration of dexamethasone likely influences the severity and short-term persistence of croup symptoms, as evidenced by less epinephrine use in the ED. Contrary to current EMS guidelines, very few patients with a pre-hospital diagnosis of croup received dexamethasone by EMS personnel. This likely represents a missed opportunity to decrease the severity of the patients' disease.

Keywords: emergency medical services (EMS), croup, dexamethasone

\section{P006}

A qualitative study of the language of satisfaction for children in the emergency department

S. Ali, MDCM, T. McGrath, MD, N. Dow, BA, S. Aziz, BSc, M. Pilarski, MD, A.L. Drendel, DO, MS; University of Alberta, Edmonton, $\mathrm{AB}$

Introduction: Measures of satisfaction are essential to understanding patient experience, and pain management. Currently, there are no validated tools to quantify children's satisfaction. To develop such a tool, we must first understand which words children use to communicate satisfaction. Our objectives were to (A) to identify the words commonly used by children of different ages to communicate satisfaction, in general, and in the context of pain management, and (B) to determine if this vocabulary is similar to that used by their caregiver. Methods: A qualitative study of 105 children-parent pairs, aged 3-16 years, who were evaluated at a pediatric emergency department (PED) from JulyNovember 2014 was conducted. Children were interviewed using a semi-structured format of ten open-ended questions. They were asked to describe their feelings when 1) they received something they wanted/ needed, 2) their expectations were met or not met in the ED, and 3) their pain was or was not relieved. A written survey was also completed by the caregiver. Interviews were transcribed and grounded theory was employed for data coding and analysis. Results: 105 child interviews were completed $(\mathrm{n}=53$ female, mean age 9.91 SD 3.71, age range 4-16). 105 caregiver surveys were completed ( $\mathrm{n}=80$ female). "Good", "better," and "happy" were most commonly used by all children $(\mathrm{n}=99)$ to express satisfaction with pain management $(27 \%, 21 \%$ and $22 \%$, respectively), with PED care $(31 \%, 14 \%$ and $33 \%)$ and in general $(13 \%, 5 \%$ and $49 \%)$. Children $(n=99)$ used the words "sad", "bad," and "not good" to communicate dissatisfaction with pain management (21\%, $7 \%$ and $11 \%$ respectively), and with PED care $(21 \%, 13 \%$ and $12 \%$, respectively). Only $55 \%$ of children understood the meaning of the word 'satisfaction'. Children used words that were similar to their caregiver $14 \%$ of the time. Conclusion: The word "satisfaction" should not be used to communicate with children in the emergency department, as many lack understanding of the term. The vocabulary that children use to describe satisfaction does not largely vary with context and involves simpler words than their parents. Caregiver vocabulary should not be used as a surrogate for pediatric patients. This study will inform the development of a validated tool to measure children's satisfaction with pain management.

Keywords: children, pain, satisfaction

\section{P007}

Association between serum biomarkers and frailty level in seniors with minor injurys

N. Allain-Boulé, MSc, J. Lebon, Ph.D, M. Sirois, PhD, M. Aubertin-Leheudre, PhD, M. Émond, MD, MSc; Centre de Recherche du CHU de Québec, Québec City, QC

Introduction: Frailty is associated with mobility \& physical impairment in seniors with minor injuries. Serum biomarkers have also been 